\title{
ON THE LATTICE OF SUBALGEBRAS OF A BOOLEAN ALGEBRA
}

\author{
G. GRÄTZER, K. M. KOH AND M. MAKKAI ${ }^{1}$
}

\begin{abstract}
The lattice of all subalgebras of a Boolean algebra is characterized.
\end{abstract}

1. Introduction. The following three facts are known: The lattice $S(\mathfrak{A})$ of all subalgebras of an algebra $\mathfrak{A}$ can be characterized as an algebraic lattice (G. Birkhoff and O. Frink, Jr. [2]), and the finitely generated subalgebras are characterized in this lattice as compact elements; if $\mathfrak{U}$ is the $2^{n}$-element Boolean algebra, then $S(\mathfrak{U})$ is dually isomorphic to the partition lattice on $n$ objects (G. Birkhoff [1]); a finitely generated Boolean algebra is finite. Combining these three facts we can conclude that the lattice $L=S(B)$ of all subalgebras of a Boolean algebra has the following property: (P) $L$ is an algebraic lattice and for each compact element $a$ of $L,(a]$ is dually isomorphic to a finite partition lattice.

Let $L$ be a lattice having this property (P). Then, with each compact element $a$ of $L$ we can associate a finite Boolean algebra $B(a)$ such that $S(B(a)) \cong(a]$. Thus, if $a$ and $b$ are compact elements of $L$ with $a \leqq b$, there is a natural embedding $\varphi_{a b}$ of $S(B(a))$ into $S(B(b))$ such that $S(B(a)) \varphi_{a b}$ is a principal ideal of $S(B(b))$. We are going to show that there is a unique embedding $\psi_{a b}$ of $B(a)$ into $B(b)$ which induces the embedding $\varphi_{a b}$. The $B(a)$ with the $\psi_{a b}$ form a direct family of Boolean algebras. We prove that if $B$ is the direct limit of this family, then $S(B)$ is isomorphic to $L$.

This result has two corollaries. Firstly, property $(\mathrm{P})$ characterizes the lattice of all subalgebras of a Boolean algebra; secondly, $S(B)$ determines $B$ up to isomorphism.

The second of these results is due to D. Sachs [6] and N. D. Filippov [3]. The first author also gave a characterization of $S(B)$ as a subsystem of an infinite partition lattice.

We refer to [4] and [5] for the undefined concepts, notations, and basic results.

Received by the editors January 11, 1972.

AMS 1970 subject classifications. Primary 06A40; Secondary 08A15, 02J05, 08A10.

Key words and phrases. Algebraic lattice, Boolean algebra, direct family, direct limit, partition lattice.

1 The research of the three authors was supported by the National Research Council of Canada.

(c) American Mathematical Society 1972 
2. Basic lemmas. To start with, we develop a series of lemmas on which our proof of the main result is based. Our main purpose is to obtain some information on the relationship between two finite Boolean algebras $A$ and $B$ provided $S(A)$ and $S(B)$ are suitably related.

Lemma 1. Let $A$ be a finite Boolean algebra. Let $p \in A, p \neq 0,1$. Then $p$ or $p^{\prime}$ is an atom of $A$ if and only if $|[p] \vee[x]| \leqq 8$, for each $x \in A$.

PROOF. $p$ or $p^{\prime}$ is an atom of $A$ if and only if for every $x$, one of $x \wedge p, x^{\prime} \wedge p, x \wedge p^{\prime}, x^{\prime} \wedge p^{\prime}$ is 0 which, in turn, is equivalent to the fact that the number of atoms of $[p] \vee[x]$, which are among the elements named, is at most three, that is, $|[p] \vee[x]| \leqq 8$.

By means of this lemma, the following result can be established.

LemMa 2. Let $A$ and $B$ be finite Boolean algebras. Let $\varphi: S(A) \rightarrow S(B)$ be a one-to-one homomorphism such that $S(A) \varphi$ is a principal ideal of $S(B)$. Then, for each atom $x$ of $A$, there is an atom $\bar{x}$ of $A \varphi$ such that $[x] \varphi=[\bar{x}]$ in $A \varphi$.

Proof. Let $x$ be an atom of $A$. Since $S(A) \varphi$ is a principal ideal of $S(B)$, there exists $b \in A \varphi, b \neq 0,1$, of $A \varphi$ such that $[x] \varphi=[b]$. Let $t$ be an arbitrary element of $A \varphi$. Then $[t]=[k] \varphi$ for some $k \in A$. Observe that, as $x$ is an atom of $A,|[x] \vee[k]| \leqq 8$ by Lemma 1 . Thus $|[b] \vee[t]|=|[x] \varphi \vee[k] \varphi|=$ $|([x] \vee[k]) \varphi| \leqq|[x] \vee[k]| \leqq 8$. Hence, by Lemma 1 again, either $b$ or $b^{\prime}$, say $b$, is an atom of $A \varphi$. Let $\bar{x}=b$. We then have $[x] \varphi=[\bar{x}]$, proving Lemma 2.

REMARK. The correspondence $x \rightarrow \bar{x}$ is unambiguous if $A$ has more than four elements.

We now associate with the map $\varphi: S(A) \rightarrow S(B)$ a map $\psi: A \rightarrow B$ in the following natural way. Let $a \in A$. Then $a=\bigvee\left(p_{i} \mid 1 \leqq i \leqq n\right)$, where for each $i=1, \cdots, n, p_{i}$ is an atom of $A$. Set $a \psi=\bigvee\left(\bar{p}_{i} \mid 1 \leqq i \leqq n\right)$.

It is obvious that $\psi$ is a one-to-one Boolean homomorphism of $A$ into $B$. The following lemma is crucial.

LEMMA 3. Assume the hypotheses of Lemma 2 and let $|A|>4$. Then $[a \psi]=[a] \varphi$, for each $a \in A$.

Proof. If $a$ is an atom of $A$, there is nothing to prove. Thus, assume that $a=p_{1} \vee p_{2} \vee \cdots \vee p_{k}$, where $k>1$, and for each $i=1, \cdots, k, p_{i}$ is an atom of $A ; p_{i} \neq p_{j}$ if $i \neq j$. Let $[a] \varphi=[\bar{a}]$. Our proof will be complete if we can show that the following holds:

$$
\begin{aligned}
& \text { either } \bar{a}=p_{1} \psi \vee p_{2} \psi \vee \cdots \vee p_{k} \psi, \\
& \text { or } \quad \bar{a}^{\prime}=p_{1} \psi \vee p_{2} \psi \vee \cdots \vee p_{k} \psi .
\end{aligned}
$$

To prove this, suppose to the contrary that $\left({ }^{*}\right)$ is false. Then, since the $p_{i} \psi$ are atoms, we have that either (1) or (2) below hold. 
(1) There is an $s$ with $1 \leqq s<k$ such that $p_{1} \psi, \cdots, p_{s} \psi \leqq \bar{a}$ and $p_{s+1} \psi, \cdots, p_{k} \psi \leqq \bar{a}^{\prime}$.

(2) $p_{1} \psi \vee \cdots \vee p_{k} \psi<\bar{a}$ or $p_{1} \psi \vee \cdots \vee p_{k} \psi<\bar{a}^{\prime}$.

There are three cases:

Case 1. Either $p_{1} \psi \vee \cdots \vee p_{s} \psi=\bar{a}$ or $p_{s+1} \psi \vee \cdots \vee p_{k} \psi=\bar{a}^{\prime}$.

By symmetry, it suffices to consider the former case. If $p_{1} \psi \vee \cdots \vee p_{s} \psi=\bar{a}$, then $p_{s} \psi$ is generated by $p_{1} \psi, \cdots, p_{s-1} \psi$, and $\bar{a}$. In fact,

Thus,

$$
p_{s} \psi=\bar{a} \wedge\left(p_{1} \psi \vee \cdots \vee p_{s-1} \psi\right)^{\prime} .
$$

$$
\left[p_{s} \psi\right] \subseteq\left[p_{1} \psi\right] \vee \cdots \vee\left[p_{s-1} \psi\right] \vee[\bar{a}]
$$

Hence, by applying $\varphi^{-1}$, we have

$$
\begin{aligned}
{\left[p_{s}\right] } & =\left[p_{s} \psi\right] \varphi^{-1} \subseteq\left(\left[p_{1} \psi\right] \vee \cdots \vee\left[p_{s-1} \psi\right] \vee[a]\right) \varphi^{-1} \\
& =\left[p_{1}\right] \vee \cdots \vee\left[p_{s-1}\right] \vee[a] .
\end{aligned}
$$

Now $p_{s}$, being an atom of $A$, would be an atom of the subalgebra $\left[p_{1}\right] \vee \cdots \vee\left[p_{s-1}\right] \vee[a]$ of $A$. But the atoms of the latter are $p_{1}, \cdots, p_{s-1}$, $a^{\prime}$, and $a-\left(p_{1} \vee \cdots \vee p_{s-1}\right)\left(=a \wedge p_{1}^{\prime} \wedge \cdots \wedge p_{s-1}^{\prime}\right)$, which is a contradiction.

Case 2. $p_{1} \psi \vee \cdots \vee p_{s} \psi<\bar{a}$ and $p_{s+1} \psi \vee \cdots \vee p_{k} \psi<\bar{a}^{\prime}$.

In this case, the subalgebra $\left[p_{1} \psi, \cdots, p_{k} \psi, \bar{a}\right]$ has $k+2$ atoms, namely, $p_{1} \psi, \cdots, p_{k} \psi, \bar{a}-\left(p_{1} \psi \vee \cdots \vee p_{s} \psi\right)$ and $\bar{a}^{\prime}-\left(p_{s+1} \psi \vee \cdots \vee p_{k} \psi\right)$. Thus, by isomorphism, $\left[p_{1}, \cdots, p_{k}, a\right]$ has $k+2$ atoms, which is false, however, since $\left[p_{1}, \cdots, p_{k}, a\right]$ has, in fact, exactly $k+1$ atoms which are $p_{1}, \cdots$, $p_{k}$, and $a^{\prime}$.

Case 3. $p_{1} \psi \vee \cdots \vee p_{k} \psi<\bar{a}$ or $p_{1} \psi \vee \cdots \vee p_{k} \psi<\bar{a}^{\prime}$.

By using a similar argument as in Case 2 , it can be shown that this case is again impossible.

Hence, we conclude that either $\bar{a}=p_{1} \psi \vee \cdots \vee p_{k} \psi$ or $\bar{a}^{\prime}=p_{1} \psi \vee \cdots \vee p_{k} \psi$, which was to be shown.

Invoking Lemma 3, we arrive at the following result which provides important information on the relation between $\varphi$ and $\psi$.

Lemma 4. Assume the hypotheses of Lemma 3. Let $X \in S(A)$. Then $X \varphi=(X) \psi$.

Proof. Let $x \in X$. Then, by Lemma 3 and the fact that $\varphi$ is isotone, we have $x \psi \in[x \psi]=[x] \varphi \subseteq X \varphi$. Thus, $(X) \psi \subseteq X \varphi$.

Conversely, let $y \in X \varphi$. Then $[y] \subseteq X \varphi$. Since $X \varphi \in S(A) \varphi$ and $S(A) \varphi$ is a principal ideal of $S(B)$, we have $[y] \in S(A) \varphi$. Hence, we have $[y]=$ $[x] \varphi$, for some $[x] \in S(A)$. As $[x] \varphi=[y] \subseteq X \varphi$ and $\varphi$ is a one-to-one homomorphism, it follows that $[x] \subseteq X$. Thus, $x \in X$.

Now, we have, by Lemma 3 again, that $y \in[y]=[x] \varphi=[x \psi] \subseteq[X \psi]=$ $X \psi$. Hence $X \varphi \subseteq(X) \psi$. 
As a conclusion, we obtain the following result.

LEMMA 5. The map $\psi: A \rightarrow B$ is uniquely determined by $\varphi: S(A) \rightarrow S(B)$ in case $|A| \neq 4$.

Proof. By Lemma 4, it suffices to prove the uniqueness part. For $|A|=2$ the assertion is trivial. If $\psi: A \rightarrow B$ is a one-to-one Boolean homomorphism inducing $\varphi$, then the image of an atom $p$ of $A$ is an atom of $A \psi=A \varphi$; moreover, $p \psi$ must belong to $[p] \varphi=\left\{0,1, p \psi,(p \psi)^{\prime}\right\}$, which contains at most one atom of $A \psi$ if $A \psi$ has at least eight elements. Hence the image of every atom of $A$ under $\psi$ is uniquely determined, and consequently so is the image of every element of $A$.

3. Main result and applications. We are now in a position to prove the main result.

THEOREM. Let $L$ be a lattice with property $(\mathrm{P})$, and let $J(L)$ be the semilattice of its compact elements. Let $\alpha_{a}$, for $a \in J(L)$, be an isomorphism: $(a] \rightarrow S(B(a))$ for some finite Boolean algebra $B(a)$. Let $\varphi_{a b}$ for $a \leqq b, a$, $b \in J(L)$, be the mapping making the diagram of Figure 1 commutative where $i:(a] \rightarrow(b]$ is the inclusion map.

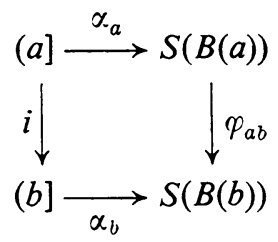

FIGURE 1

Then there is a direct family $\mathscr{B}=\left\langle B(a), \psi_{a b}\right\rangle$ such that: (1) $\psi_{a b}$ induces $\varphi_{a b}$, i.e., $(X) \psi_{a b}=X \varphi_{a b}$ for any subalgebra $X \subseteq B(a)$; (2) the $\psi_{a b}$ are uniquely determined for every $a \in J(L)$ of height $\neq 1$ (i.e., $|B(a)| \neq 4) ;(3) S(B) \cong L$ where $B$ is the direct limit of $\mathscr{B}$.

Proof. Since the diagram of Figure 1 is commutative for $a, b \in J(L)$, $a \leqq b, S(B(a)) \varphi_{a b}$ is a principal ideal of $S(B(b))$. Hence, by previous lemmas in $\S 2$, there is a one-to-one Boolean homomorphism $\psi_{a b}: B(a) \rightarrow B(b)$ which induces $\varphi_{a b}$. By Lemma 5 , the $\psi_{a b}$ are uniquely determined if $|B(a)| \geqq 8$. From this and from the fact that $\varphi_{a b} \varphi_{b c}=\varphi_{a c}$, for $a \leqq b \leqq c$, it follows that $\psi_{a b} \psi_{b c}=\psi_{a c}$ if $a \leqq b \leqq c$ and $|B(a)| \neq 4$.

Since the uniqueness of $\psi_{a b}$ inducing $\varphi_{a b}$ fails for $a \in J(L)$ of height 1 , we have to be a little careful in defining $\psi_{a b}$ for $a$ of height 1. Let $a$ be an element of $J(L)$ of height 1 . If there is no $b \in J(L)$ with $b>a$ then there is nothing to prove since then $L$ is the lattice of subalgebras of a four element 
Boolean algebra. Otherwise choose $\bar{a} \in J(L)$ covering $a$, i.e. $a<\bar{a}, \bar{a}$ is of height 2. Select $\psi_{a \bar{a}}: B(a) \rightarrow B(b)$ inducing $\varphi_{a \bar{a}}$ (there are, in fact, two possibilities for $\left.\psi_{a \bar{n}}\right)$. Let $\psi_{a a}$ be the identity on $B(a)$. Now, for any $b>a$, let $c=b \vee \bar{a}$ and define $\psi_{a b}$ such that the diagram of Figure 2 commutes.

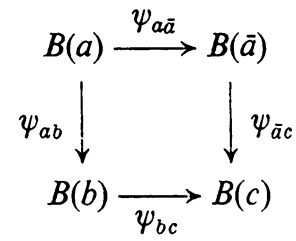

FIGURE 2

Notice that $X=B(a) \psi_{a \bar{a}} \psi_{\bar{a} c}=B(a) \varphi_{a c}=a \alpha_{c} \subseteq B(c)$, and $Y=B(b) \psi_{b c}=$ $B(b) \varphi_{b c}=b \alpha_{c} \subseteq B(c)$; hence $X \subseteq Y$ and thus $\psi_{b c}^{-1}$ is defined on $X$, i.e. $\psi_{a b}$ can indeed be determined in a unique way. It easily follows that $\psi_{a b}$ induces $\varphi_{a b}$. Having defined $\psi_{a b}$ for any $a \leqq b$ in $J(L)$, the transitivity $\psi_{a b} \psi_{b c}=\psi_{a c}$ is easily checked for any $a \leqq b \leqq c$ in $J(L)$. Since $J(L)$ is a join semilattice and hence is a directed set, we have a direct family $\mathscr{B}=\left\langle B(a), \psi_{a b}\right\rangle$ of finite Boolean algebras. Let $B$ be the direct limit of $\mathscr{B}$. We shall prove that $L \cong S(B)$. Since $L$ is generated by $J(L)$, to define a map from $L$ to $S(B)$, we define $f: J(L) \rightarrow S(B)$ as follows: for $x \in J(L)$ set $f(x)=B(x) \psi_{x \infty}$.

Note that as $\psi_{a b}$ is a one-to-one Boolean homomorphism for all $a \leqq b$, $a, b \in J(L), \psi_{x \infty}$ is a one-to-one Boolean homomorphism for each $x \in J(L)$.

We claim that, if $x, y \in J(L)$, then $x \leqq y$ if and only if $B(x) \psi_{x \infty} \subseteq B(y) \psi_{y \infty}$.

To see this, let $x, y \in J(L)$. Then there exists $u \in J(L)$ such that $x, y \leqq u$. By means of the commutative diagram of Figure 1, it follows that $x \alpha_{x} \varphi_{x u}=x i \alpha_{u}$, i.e.,

$$
x \alpha_{u}=B(x) \varphi_{x u}=B(x) \psi_{x u},
$$

by Lemma 5 . Similarly,

$$
y \alpha_{u}=B(y) \psi_{y u} .
$$

Now, observe that $x \leqq y$ :

if and only if $x \alpha_{u} \subseteq y \alpha_{u}$,

if and only if $B(x) \psi_{x u} \subseteq B(y) \psi_{y u}$,

if and only if $B(x) \psi_{x u} \psi_{u \infty} \subseteq B(y) \psi_{y u} \psi_{u \infty}$,

if and only if $B(x) \psi_{x \infty} \subseteq B(y) \psi_{y \infty}$,

which was to be shown.

Hence, $f$ is a one-to-one order- and inverse order-preserving map.

Let $A$ be a finite subalgebra of $B$. Then there is $u \in J(L)$ such that $A$ is a subalgebra of $B(u) \psi_{u \infty}$. Thus, $A \psi_{u \infty}^{-1}$ is a subalgebra of $B(u)$. Hence $A \psi_{u \infty}^{-1}=B(x) \psi_{x u}$ for some $x \in J(L), x \leqq u$. Observe that

$$
f(x)=B(x) \psi_{x \infty}=B(x) \psi_{x u} \psi_{u \infty}=A \psi_{u \infty}^{-1} \psi_{u \infty}=A \text {. }
$$


Therefore, we conclude that $f$ is an isomorphism of $J(L)$ onto the semilattice of compact elements of $S(B)$ and hence can be extended to an isomorphism of $L$ onto $S(B)$. The proof of the theorem is thus complete.

The following are two applications of the main result.

COROLlARY 1. The lattice $S(B)$ of all subalgebras of a Boolean algebra $B$ can be characterized as a lattice satisfying property $(\mathrm{P})$.

Corollary 2 (D. SACHS). The lattice $S(B)$ determines the Boolean algebra $B$ up to isomorphism.

Corollary 1 follows immediately from the main theorem. To prove Corollary 2 , let us say that a direct family $\left\langle B_{a}, \psi_{a b}\right\rangle, a \leqq b, a, b \in J(L)$, is associated with $L$ if it satisfies the conclusion of the theorem with some system $\left(\alpha_{a}\right), a \in J(L)$, of isomorphisms $\alpha_{a}:(a] \rightarrow S\left(B_{a}\right)$. It easily follows from the uniqueness part of the theorem that any two direct families associated with $L$, and hence their direct limits too, are isomorphic. Now, let $L=S(A)$ for some Boolean algebra $A$. Consider the inclusion maps $\psi_{a b}: a \rightarrow b$ for $a \subseteq b, a, b$ subalgebras of $A$. Then the system $\left\langle a, \psi_{a b}\right\rangle$ is associated with $L$, in fact with the $\alpha_{a}$ being identity mappings. Moreover, the direct limit of $\left\langle a, \psi_{a b}\right\rangle$ is isomorphic to $A$. As a conclusion, we have that for $L \cong S(A), A$ a Boolean algebra, some direct family associated with $L$ has a direct limit isomorphic to $A$. If we have $S(A) \cong S(B)$, then the last assertion applied twice to $L=S(A)$ gives direct families associated with $L$ with direct limits isomorphic to $A$ and $B$, respectively, hence $A \cong B$.

\section{REFERENCES}

1. G. Birkhoff, Lattice theory, 2nd. rev. ed., Amer. Math. Soc. Colloq. Publ., vol. 25, Amer. Math. Soc., Providence, R.I., 1948. MR 10, 673.

2. G. Birkhoff and O. Frink, Jr., Representations of lattices by sets, Trans. Amer. Math. Soc. 64 (1948), 299-316. MR 10, 279.

3. N. D. Filippov, Projectivity of lattices, Mat. Sb. 70 (112) (1966), 36-54; English transl., Amer. Math. Soc. Transl. (2) 96 (1970), 37-58. MR 33 \#5527.

4. G. Grätzer, Universal algebra, University Series in Higher Math., Van Nostrand, Princeton, N.J., 1968. MR 40 \#1320.

5. - Lattice theory: First concepts and distributive lattices, Freeman, San Francisco, Calif., 1971.

6. D. Sachs, The lattice of subalgebras of a Boolean algebra, Canad. J. Math. 14 (1962), 451-460. MR 25 \#1116.

Department of Mathematics, University of Manitoba, Winnipeg, Manitoba, CANADA 